\title{
Displaying Laterally Moving Tactile Information
}

\author{
Joerg Fricke, Helmut Baehring \\ Department of Computer Science, FernUniversitaet Hagen \\ D-58084 Hagen, Germany \\ e-mail joerg.fricke@fernuni-hagen.de
}

\begin{abstract}
A movable dynamic tactile display has been developed presenting information to one or several fingertips resting on the display. As a main goal, the virtual line or plane of information to be displayed should be read using the same perceptual and cognitive resources as with real objects like paper braille or tactile graphics. This is achieved by a nonvibratory display comprising an array of tactile pins having a spacing of about $1 \mathrm{~mm}$, at least in the x-direction. These pins can be lifted by piezoelectric bending strips to a variety of heights. A scanned braille dot, for example, is presented by a smooth lateral "wave" of lifted and lowered pins. A special design resulting in a negligible power dissipation even at high bandwidth allows the integration of the bending strip drivers into the display mechanics. First experiences with a prototype are reported, and future research topics are outlined.
\end{abstract}

\section{Introduction}

For blind or visually impaired computer users, dynamic tactile output devices are useful besides speech output, depending on the application and on the preferences of the user. Surely, the most convenient solution is to provide a real line resp. a real screen of braille or graphics. However, if size, weight, or costs are critical factors, then a virtual line or a virtual screen presented only partially at a time by a small movable display could be a satisfactory compromise. This is especially true if the information to be read does not contain complex graphics but text enhanced by a limited set of graphic symbols and providing some relevant information by its twodimensional structure, as the mathematical notation Dotsplus [1], for example.

Virtual objects displayed by movies to the visual sense can be recognized without training. This is due to the fact that looking at a movie screen involves the same perceptual and a subset of those cognitive resources which are engaged in looking at real world objects. Our goal was to develop an equivalent display for the tactual sense, for the present restricted to the presentation of braille characters to one or a few fingertips. Each braille reader should be able to use this device achieving his or her normal reading performance without any special training. Such a dynamic braille display can be mounted on a laterally moveable carriage [2] or on some kind of mouse similar to the Optacon puck [3].

To outline the background, section 2 presents some fundamentals on tactual signal processing, and section 3 gives a survey on state-of-the-art devices. After introducing 
the principles of our "Braille Movie" approach in section 4, we describe the features of the laboratory devices in section 5. Section 6 presents first experiences made by informal testing of a prototype, section 7 deals with the design of devices comprising a Braille Movie display, and section 8 is concerned with future research topics.

\section{Perceptive and cognitive processing of tactile signals}

Braille and tactile graphics can be considered as texture providing information by a spatial structure. For this reason, many results of research on texture perception apply as well to tactual reading [4]. In the skin of the fingertips, there are receptors responding to perpendicular indentation of the skin (SA I), to tangential displacement by friction (SA II), to vibration in the range of 5 to $40 \mathrm{~Hz}$ (RA), and to vibration in the range of 40 to $400 \mathrm{~Hz}$ (PC) [4]. In spite of the fact that scanning a rough surface should excite always SA I and SA II units and, depending on the surface pattern and the scanning speed, RA or PC units as well, it was shown that lowering the response of the SA II, RA, or PC units does not affect the perception of roughness [5, 6]. The respective cognitive process seems to take only, or mainly, the SA I signals into account. Since the SA I units allow also a sufficient localization of the stimuli, tactile structures like braille can be read without any friction and within a wide range of scanning speed. Even more, the absense of friction as a source of "noise" should improve the reading performance [7].

Although Braille readers commonly use several fingers of both hands in order to track the current and the following line and to explore in advance the structure of the text, e.g. the length of words and sentences, the actual reading is performed by just one finger of one or both hands each [8].

Navigating through a complex text is eased by dealing with a mental image of the text structure, and constructing such images is facilitated by some proprioceptive feedback reporting the position of the hand during the reading.

To summarize, "natural" tactile reading involves at least the stimulation of the SA I receptors by indentation, rather of several fingertips than of only one, and some proprioceptive feedback concerning the position of the reading fingers.

Vibrotactile displays offer an advantage mainly on the engineering side: The PC units can be stimulated by skin displacements of a few $\mu \mathrm{m}$, i.e. by transmitting a small amount of energy. However, the user has to develop by training a pattern recognition process based on the PC signals. For this reason, results obtained by experiments based on vibrotactile displays apply not, or only partially, to braille reading and dealing with static tactile graphics.

The spatial resolution of the skin of the fingertips is different depending on whether recognition, localization, or distinction of perpendicularly tapped or tangentially scanned tactile stimuli are concerned: A single point stimulus can be localized with an accuracy of about $1 \mathrm{~mm}$. Two simultaneous tactile point stimuli are felt by tapping as two distinct objects if their distance is larger than about $2 \mathrm{~mm}$. Structure widths of $0.1 \mathrm{~mm}$ are well perceived by scanning the object. Likewise, a movement is already recognized if a single stimulus is moving laterally for about $0.1 \mathrm{~mm}$ [9]. 
Consequently, a braille dot or a raised line composed of several narrow dots or pins is felt as a single object when touched without tangential movement but its rough structure is perceived by scanning. A slow sequence of lateral "jumps" of an tactile object can be felt as a smooth motion if the width of each jump is only a fraction of $1 \mathrm{~mm}$.

As for the visual sense, one apparently moving object can be perceived if two stimuli are applied to distant points of the skin sequentially, but it feels different from an actually moving stimulus [10] - perhaps due to the high temporal resolution of the tactual sense.

Due to the adaptation of the SA I units, the structure of complex static information is well recognized only by scanning. Additionally, it is reasonable to assume that the determination of the structure is based not on the SA I signals itself but on their changes, i.e. on the movements of the edges of the indentations. In this case, tapping on a structure or popping up some information under the resting finger provide a perceptual pattern that did not match any pattern out of the temporal sequence obtained by scanning the same structure because the progressive indentation during the first phase of tapping causes only "leading" but no "trailing", i.e. releasing edges, and the edges move perpendicularly to their own local tangent instead of unidirectionally on the whole fingertip as during scanning.

\section{State-of-the-art devices}

There are some proposals how to display moving braille characters by a wheel [11] or by a belt [12] carrying balls or pins inserted or removed by an electromechanical display controller. Certainly, such devices would provide the same sensations as static braille does but they would be too heavy and too bulky to be integrated in a mouse, for example. The well known vibrotactile Optacon display [13] is not designed to show laterally moving Braille characters but enlarged ink print characters and graphics. Of course, with a modified pin matrix geometry it could do so but even a skilled braille reader would have to learn to process the vibrotactile sensations. Furthermore, some devices displaying a virtual braille line contain one or a few usual braille cells [2], [14]. During scanning the line, the characters are "popped up". Again, the user has to undergo some training because instead of being provided with the usual temporal sequence of spatial patterns he has to recognize a single unfamiliar pattern, as mentioned in section 2 .

\section{Providing the impression of moving dots}

Obviously, laterally moving tactile objects on a horizontal surface can be obtained by transversale waves, i.e. by lifting and lowering parts of the surface in an appropriate sequence without moving any material laterally. Smoothly moving very small pieces of the surface would result in a virtually noisefree data presentation to the skin resting on the surface. The distinctness of the perception would be better than by scanning 
the static information by the skin. By choosing a coarse partitioning of the surface and by limiting the number of intermediate levels of the surface parts, the display becomes producable and affordable but some noise is introduced. Fortunately, braille readers and users of static tactile graphics are accustomed to a certain amount of noise by scanning the surface with the skin. So, the noise should be limited just to the "normal" level.

While the static spatial resolution of the display is given only by the partitioning of the surface, the dynamic spatial resolution, i.e. the number of steps the information is doing to move along a given distance, is determined by the distance between the surface parts and by the number of heights the parts of the surface can be driven to.

Laterally moving 8 dot braille characters results in 4 "dot tracks". Hence a dynamic braille display can consist of a solid surface with 4 rows of vertically moveable tactile pins. In the direction perpendicular to the movement, the width of the pins should just equal the width of the presented dots. In the direction of the movement, the width of the pins can be determined by the following considerations. During the movement of a dot for one pin width, the height of the dot should not change considerably in order to yield a nonvibrating dot. This can be achieved by holding one pin at full height as long as one neighbouring pin is lifted and the other neighbour is lowered. Consequently, one dot has to be presented by at least two or three pins depending on the phase of movement. Since there must be a gap between two dots in the same row, the maximal pin width plus spacing is a third of the lateral center-to-center distance of the braille dots. Figure 1 shows the arrangement of pins in a braille display. Figure 2 is a side-view of pins presenting two moving dots in four phases. The shown dot spacing of $3 \mathrm{~mm}$ could be enlarged to improve the separation of neighbouring dots.

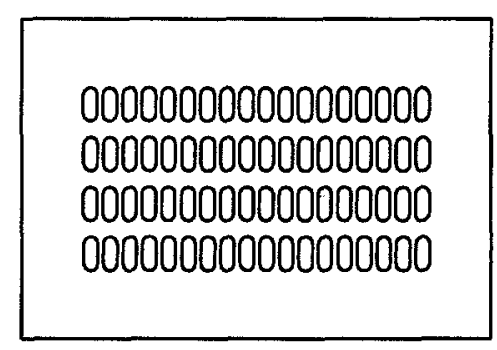

Fig. 1. The arrangement of pins in a braille display surface (drawing enlarged)

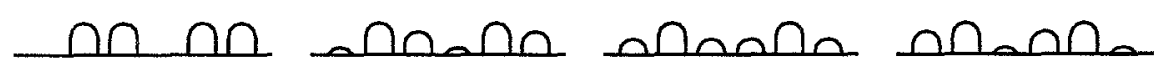

Fig. 2. $1 \mathrm{~mm}$ spaced pins displaying two braille dots moving laterally by steps of $0.25 \mathrm{~mm}$

Graphic displays should comprise a pin array having the same spacing in $\mathrm{x}$ - and $\mathrm{y}-$ direction. On principle, presenting graphics using a frictionless display as described could lead to a difficulty: a long horizontal or vertical stroke would perceptually 
vanish during a scanning movement parallel to its axis because the contents of the display would not change. Of course, the display controller could recognize such situations and introduce small movements of the display contents perpendicular to the main movement. However, having a sufficiently high dynamic spatial resolution, this problem will virtually never arise because, as during scanning real structures, the user will never carry out exactly straight movements. As long as the amplitude of the wavy line he moves along is larger than half the spatial quantization caused by the dynamic resolution, the display contents will vary and the stroke will stay perceivable.

Both braille and graphic displays of the kind described above have a peculiarity: With the fingertips resting on the moving display, the scanned objects are felt as smooth and the tactile pins itself are not distinguishable. However, if the user starts to scan the display surface the smooth objects turn into rough ones because the gaps between the pins becomes perceivable although not localizable.

\section{The first laboratory displays ("Braille Movies")}

Till now, two displays are built. The first display contains 4 rows of 18 pins each. The center-to-center distance of neighbouring pins is $1 \mathrm{~mm}$ in lateral direction and $2.5 \mathrm{~mm}$ from row to row. The horizontal cross-section of each pin is nearly rectangular with a size of about $0.7 \mathrm{~mm}$ by $1.7 \mathrm{~mm}$. So, a braille dot represented by two adjacent pins has a basis of 1.7 by $1.7 \mathrm{~mm}$. The angles on top of the pins are smoothed. The geometry of the second display differs in having a row-to-row spacing of $3 \mathrm{~mm}$, the remaining measurements equal those of the first display.

Each pin is driven by one piezoelectric bending strip. In order to provide sufficient forces and to match the distance of $1 \mathrm{~mm}$, the strips being arranged in three layers for each row of pins have a width of $2.5 \mathrm{~mm}$ but narrow ends of $0.8 \mathrm{~mm} .12$ layers of bending elements are glued within a stack of 13 printed circuit boards (PCBs).

In the second version, the 11 inner PCBs of the stack are replaced by two thinner PCBs each resulting in a decomposable stack of 12 modules. As shown in figure 3 , each module carries also the electronic drivers of the piezoelectric benders and a shift register. Both the volume and the profile of the display including these drivers match approximately that of an assembly of 4 usual braille cells.

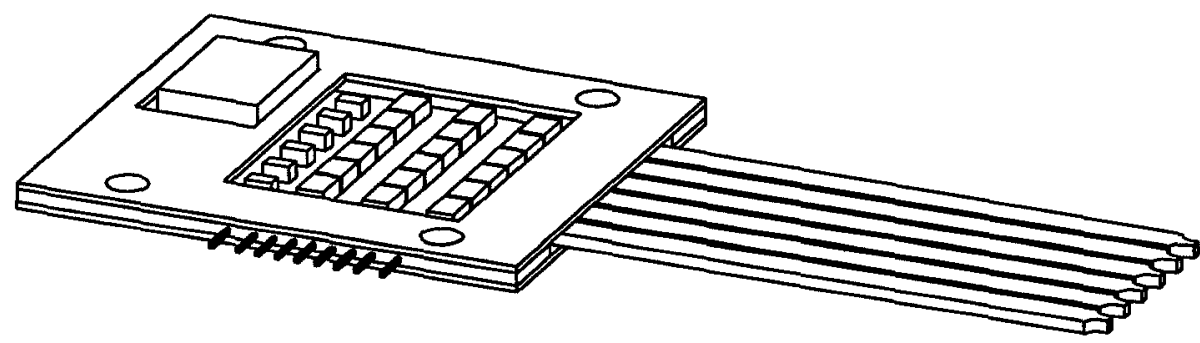

Fig. 3. A perspective view of a module carrying bending strips, drivers, and a shift register 
The capacitances of the piezoelectric benders are not switched to one out of two DC supply voltages by means of open collector or push-pull output stages, as usually. Instead, in order to achieve a low power dissipation within the drivers even during fast movements of the displayed information, the electrodes are charged from a sinusshaped $\mathrm{AC}$ voltage of up to $500 \mathrm{~Hz}$ by means of controlled halfbridge rectifiers. Given a reading speed of 14 characters per second, a carriage return time of 1 second, characters with 3 dots on an average, a capacitance of $40 \mathrm{nF}$ per bending strip, and a control voltage range of $200 \mathrm{~V}$, we had an average heat dissipation within the 72 compactly arranged drivers of about $6 \mathrm{~W}$ with push-pull outputs but we have less than $0.1 \mathrm{~W}$ with controlled rectifiers. Each electrode can be charged to an arbitrary value within the voltage range of the $\mathrm{AC}$ voltage by changing the state of the corresponding driver each time the $\mathrm{AC}$ voltage reaches the desired value. So, an arbitrary force within the given range can be exerted on each individual tactile pin.

A microcontroller on an additional PCB is executing the following tasks: Communications with the attached personal computer using a serial interface, determining the position of the display unit and hence the section of the virtual braille line to be displayed, conversion of the supply voltage of $5 \mathrm{~V} \mathrm{DC}$ to $200 \mathrm{~V} \mathrm{DC}$ and to $200 \mathrm{Vpp} \mathrm{AC}$ by means of switching converters, and controlling the rectifiers which are driving the bending strips. So, except for the geometry of the display, the way the text is displayed may be altered rather easily by software changes.

\section{First experiences testing the Braille Movie}

We presented the prototype (first version) during an exhibition. No questionnaire nor any schematic way to process the testing of the display by the visitors of the exhibition was prepared. So we just gave some hints to the visitors how to use the device, and the persons testing the display reported in an informal way the recognized characters and words and the difficulties they discovered during reading. Obviously, such a procedure cannot yield any numerical results but first impressions and hints about which problems should be faced during the next steps of the development.

About 50 persons tried to read using the Braille Movie. Two stated that they would refuse to use such a device. About $95 \%$ succeeded in reading the single characters and words presented by the test programs. Their general attitudes were in the range between relevant criticism and enthusiasm. Topics critized by many were:

a) There was some tactile noise in the background, i.e. though the display should be nonvibrating sometimes some pins were actually vibrating.

b) Neighbouring dots appeared not distinctly separated from each other, i.e. a "C" could be taken for a horizontal stroke, for example.

c) Braille dots 2 and 5 were perceived weaker than the rest of the dots.

Topic a) was due to a software bug we could not fix before the beginning of the exhibition. Of course, this is not an inherent problem.

b) should be solved or at least considerably improved by changing the shape of the pins. Using pins with more narrow ends in the direction of the information movement, the gaps between neighbouring dots will be better perceivable. Furthermore, as 
mentioned in section 4 , the horizontal distance of the braille dots can be enlarged. The consequently enlarged width of the virtual line can be compensated by moving the virtual line contrary to the direction of the movement of the display unit [2].

It seems rather unlikely that problem c) was caused by generally weaker bending strips in the second row. Perhaps this is a general effect with this kind of displays. We have enlarged the row-to-row distance to $3 \mathrm{~mm}$ in the second display in order to avoid dots 2 and 5 to be hidden by the other dots.

Having in mind that this was the very first approach and that the display is certainly improvable, we feel that the basic principle will become a sufficiently convenient way to display Braille when costs or size and weight of the output device are critical factors.

\section{Possible features of devices containing a Braille Movie}

To provide some proprioceptive feedback, a device presenting a virtual screen can be designed. as a free moveable unit like a mouse or with the display mounted on a guided carriage. A mouse would be less bulky and more lightweight and allows arbitrary wide movements, on principle. However, its use requires a flat support, the user has to care for not confusing the cable, and tracking lines of characters has to be supported by acoustic or tactile signals. A mouse employing an optical position detector running on a pad with a coarse pattern would provide a virtually errorfree absolute modus, like a digitizing tablet. However, an optical mouse is smaller, less weighty, and less expensive than a tablet. The working area can be easily adapted to the current application either by changing the pad or by folding it like the board of a board game. A large but foldable pad can well be employed with portable equipment. A device guiding a carriage is easy to use and provides space for additional control knobs and keys. It could be placed partially under the keyboard like conventional braille lines. An unusual but promising approach could be a "flying mouse" on a virtual pad. That could be achieved by carrying the mouse by some rods with angle encoders and electric controlled brakes at each joint of the rods.

Generally, some coarse tactile elements on the right of the high resolution display would allow a prerecognition of the word boundaries. Besides functions supporting the navigation and orientation in structured texts and tables [2], features concerned with reading plain texts could be valuable, too. For example, a fast movement of the display to the left end of the line could cause an automatic line feed. In this way, a large text could be read just by moving the display.

\section{Future research topics within the Braille Movie approach}

The Braille Movie render possible various display modi. For example, the spacing between dots, characters, and words can be varied, different attributes of characters can be indicated by different low vibrating frequencies, and the presented virtual braille line can be resting or moving contrary to the movement of the display unit. 
The reading performance of both skilled and unskilled braille readers should be compared using paper braille, conventional braille lines, and a Braille Movie with combinations of the mentioned display modi. Learning braille using a Braille Movie from the beginning could be advantageous because it may help to concentrate on just one fingertip, and since the computer can track the reading finger a teaching system could provide some individual help by speech.

Also, it would be interesting to display ink print characters and plain graphical elements using a "Graphic Movie". Compared to vibrotactile displays, we expect the users to show a similar performance but to become skilled after a considerably shorter period of training.

\section{References}

1. W.A. Barry, J.A. Gardner, T.V. Raman: Accessibility to Scientific Information by the Blind: Dotsplus and ASTER could make it easy. To be published in: Proceedings of the 1994 CSUN Conference on Technology and Person with Disabilities, Los Angeles 1994

2. A. Parreno, P.J. Magallon: Teresa'80: An 80 Character Single Cell Braille Line. In: W. Zagler (ed.): Computers For Handicapped Persons. Proceedings of the 3rd Int. Conference, Vienna 1992, pp. 403-408

3. G. Vanderheiden et. al.: A dual information class model for providing access to computers with graphic user interfaces for people who are blind. Proceedings of The First World Conference on Information Technology, Washington D.C. 1991, Vol. III, pp. 77-89

4. S.J. Lederman: The perception of texture by touch. In: W. Schiff, E. Foulke (eds.): Tactual perception. Cambridge University Press 1982, pp. 142-155

5. M.M. Taylor, S.J. Lederman: Tactile roughness of grooved surfaces: A model and the effect of friction. Perception \& Psychophysics 17 (1), 23-36 (1975)

6. S.J. Lederman, J.M. Loomis, D.A. Williams: The role of vibration in the tactual perception of roughness. Perception \& Psychophysics 32 (2), 109-116 (1982)

7. S.J. Lederman: "Improving one's touch" $\ldots$ and more. Perception \& Psychphysics 24 (2), 154-160 (1978)

8. J.S. Lappin, E. Foulke: Expanding the tactual field of view. Perception \& Psychophysics 14 (2), 237-241 (1973)

9. J.M. Loomis, C.C. Collins: Sensitivity to shifts of a point stimulus: An instance of tactile hyperacuity. Perception \& Psychophysics, Vol. 24 (6), 487-492 (1978)

10. C.E. Sherrick, R. Rogers: Apparent haptic movement. Perception \& Psychophysics 1, 175-180 (1966)

11. German patent application DE 3033078 (1980)

12. European patent application EP 0123205 (1984)

13. J.C. Bliss, M.H. Katcher, C.H. Rogers, R.P. Shepard: Optical-to-Tactile Image Conversion for the Blind. IEEE Transactions on Man-Machine Systems, Vol MMS-11 (1), 58-65 (1970)

14. J.T. Ohsann: New role for 'mice'. IBM Research Magazine (publ. date unknown) 